\title{
Perils of petrotectonic modeling: A view from southern Sonora, Mexico
}

\author{
Christy B. Till *, Phillip B. Gans, Frank J. Spera, Ian MacMillan ${ }^{1}$, Karen D. Blair ${ }^{2}$ \\ Department of Earth Science, University of California, Santa Barbara, CA 93106, United States
}

\section{A R T I C L E I N F O}

\section{Article history:}

Received 12 February 2009

Accepted 30 June 2009

Available online $\mathrm{xxxx}$

\section{Keywords:}

petrotectonic models

Sonora, Mexico

Gulf of California

subduction

oblique extension

pre-eruptive water content

\begin{abstract}
A B S T R A C T
We present major and trace element geochemical data from well-dated Oligocene-Miocene volcanic sections in southern Sonora, Mexico $\left(28^{\circ}, 110^{\circ}\right)$ that span the transition from subduction to oblique extension in the proto-Gulf of California. The region of northwest Mexico including Sonora experienced this abrupt change in tectonic setting ca. 15-12.5 Ma. Syn- and post-subduction calc-alkaline volcanic rocks erupted in Sonora all have similar major and trace element signatures indicative of a continental arc setting. Post-subduction rocks in Sonora document only a subtle change in the behavior of high field strength, large ion lithophile and rare earth elements and a decrease in pre-eruptive water-content. The protracted geochemical changes in Sonora suggest it took at least 4 m.y. to erase the subduction signature from the sub-arc mantle here. Therefore Sonoran geochemistry is not consistent with the predictions of petrotectonic models and contrasts with central and southern Baja where a rapid and distinct change in the composition of lavas erupted is reported synchronous with the tectonic transition. This study exemplifies the need for caution when interpreting geochemical data for tectonic information in the absence of plate reconstructions.
\end{abstract}

(C) 2009 Elsevier B.V. All rights reserved.

\section{Introduction}

The use of petrochemistry to identify the tectonic setting in which magmas were formed can be traced back to the work of Marshall (1912) and Daly (1933) or in the modern era to the work of Chayes (1964) and Pearce and Cann $(1971,1973)$. These authors demonstrated that the trace element concentrations of basalts varied with known tectonic settings and could be used to identify paleotectonic environments. However, Pearce and Cann acknowledged the need for greater understanding of trace element partitioning and how it might have changed during Earth's history. Since the 1970's our understanding of trace element partitioning between liquids and solids of varying composition and during crystallization and melting processes has increased dramatically. Because of these scientific advances, trace elements concentrations continue to be useful petrotectonic indicators (e.g., Weigand et al., 2002; Kovács and Szabó, 2008), especially where tectonic plate reconstructions are not accessible.

Extensive work on the character of primary subduction zone lavas generated by hydrous melting of the mantle wedge demonstrates they are on average basaltic-andesitic and have trace element signatures enriched in large ion lithophile elements (LILE) and depleted in high field strength elements (HFSE) (Perfit et al., 1980; Gill, 1981; Arculus and Johnson, 1981; Dupuy et al., 1982; Pearce and Peate, 1995). Continental

\footnotetext{
* Corresponding author. Now at Department of Earth, Atmospheric and Planetary Sciences, Massachusetts Institute of Technology, Cambridge, MA, 02139, United States. E-mail address: ctill@mit.edu (C.B. Till).

${ }^{1}$ Now at Questa Environmental Consulting, Los Angeles, CA 90027, United States.

2 Department of Physical Sciences, Houston Community College Southwest Stafford TX 77477, United States.
}

extension-related magmatism, generated by decompression melting of the anhydrous asthenosphere, has a greater variability in composition. Primary melts are in general similar to ocean island basalts (OIB) and can range from tholeiitic to alkaline olivine basalts. Extension-related magmas often erupt in bimodal basaltic and rhyolitic suites when involved in crustal anatexis (Christiansen and Lipman, 1972). Extensionrelated trace element signatures are distinguished from subductionrelated signatures by their lack of HFSE depletion. Therefore petrotectonic models would predict a corresponding change in the geochemistry of the lavas, from high to low LILE /HFSE ratios, for a region that experiences a change in tectonic setting from subduction to extension.

The Gulf Extensional Province (GEP) (Gastil et al., 1975) of northwestern Mexico is a structural province defined as the region of extended continental crust in eastern Baja California, and the northwestern part of mainland Mexico adjacent to the Gulf of California (Stock and Hodges, 1989). An extensive suite of volcanic rocks was erupted within the GEP spanning the tectonic transition from subduction to oblique extension along this portion of the North American plate margin (Gastil et al.,1979; Hausback, 1984; Oskin and Stock, 2003; Mora-Klepeis and McDowell, 2004). This suite of volcanic rocks, in conjunction with the well understood plate reconstructions for this region (Atwater, 1970; Atwater, 1989; Lonsdale, 1991, 2006), make it an ideal location for determining if the geochemical response to a change in tectonic setting from subduction to extension is similar to that expected according to simple petrotectonic models. Many geochemical surveys of syn- and post-subduction volcanism on the rifted Baja peninsula have examined how the western segment of the GEP responded to the change in tectonic setting. Similar to petrotectonic predictions, a systematic change from typical arc-related calc-alkaline volcanism to a diverse 
suite of alkalic, tholeiitic, and high-Nb basaltic volcanism, in addition to local adakite volcanism, is reported for central and southern Baja, coincident with the change in tectonic setting from subduction to rifting ca. 12.5 Ma (Gastil et al., 1979; Hausback, 1984; Sawlan and Smith, 1984; Saunders et al., 1987; Bigioggero et al., 1995; Luhr et al., 1995; NegreteAranda and Cañón-Tapia, 2008; Castillo, 2008). However little work has been done in the eastern GEP in mainland Mexico to understand how this region of thicker continental crust (Valencio-Moreno, 2001) responded geochemically to the change in tectonic setting. Hence, this study examines a suite of volcanic rocks from the eastern GEP that offer excellent age and spatial control to test the resolution of the geochemical record in reproducing the tectonic and/or structural history of a region. Here we will show that there are only second-order changes in the major and trace element geochemistry of Sonoran volcanism during the change from subduction to extension ca. 15-12.5 Ma, which is not consistent with the predictions of petrotectonic models.

\section{Tectonic and geologic background}

The region of northwest Mexico now divided into the Baja peninsula and Sonora, Mexico by the Gulf of California rift, experienced subduction of the Farallon plate for most of the Tertiary. The East Pacific Rise (EPR) entered the North American trench near the latitude of present-day Los Angeles at $25 \mathrm{Ma}$, creating the strikeslip boundary between the North American and Pacific plates (Atwater, 1970; Stock and Hodges, 1989). Continued subduction of the EPR caused this strike-slip boundary to grow in length and the cessation of the subduction zone west of Baja and Sonora to migrate south from 25-15 Ma (Dickinson and Snyder, 1979). Subduction of the EPR ceased west of Baja between 15 and 12.5 Ma, triggering the southward jump of the Rivera Triple Junction and the end of subduction west of Baja and Sonora (Lonsdale, 1991, 2006). Reconfiguration of the North American-Pacific plate boundary during the Miocene resulted in transfer of the Baja peninsula to the Pacific plate and a change in plate motion vectors from convergence to oblique divergence between 12.5 and $\sim 6 \mathrm{Ma}$.

Magmatism related to subduction of the Farallon plate occurred in Baja California and Sonora, Mexico from 100 to $45 \mathrm{Ma}$, followed by widespread silicic magmatism in the Sierra Madre Occidental from 37 to $23 \mathrm{Ma}$ (McDowell and Clabaugh, 1979). Magmatism evidently swept westward through central and western Sonora to eastern Baja from 26 to $\sim 12 \mathrm{Ma}$, as it became more intermediate to mafic in composition (Sawlan, 1991; Aranda-Gomez and McDowell, 1998; Gans et al., 2006). This syn-subduction volcanism in Sonora was diverse and diffuse, unlike a more linear Andean-type arc. Postsubduction volcanism consisted of basaltic to rhyolitic volcanism, which persisted in coastal Sonora until $\sim 8 \mathrm{Ma}$ and on Baja and Isla Tiburon until $\sim 6 \mathrm{Ma}$, synchronous with proto-Gulf deformation (Gastil et al., 1999; Oskin and Stock, 2003; Gans et al., 2006).

Eastern Sonora records an episodic history of Eocene to midMiocene volcanism, volcaniclastic sedimentation, and extensional faulting, virtually all of which predates the tectonic transition from subduction to oblique divergence (Gans, 1997; McDowell et al., 1997; Wong and Gans, 2003; Gans et al., 2006; Wong and Gans, 2008) (Fig. 1). Extension in eastern Sonora is therefore mainly "intra-arc" or "back-arc." The younger part of the volcanic record in eastern Sonora ( 22-15 Ma) overlaps in age with the oldest volcanism in coastal Sonora, which facilitates a comparison of syn-subduction volcanism in an E-W transect across Sonora. Coastal Sonora is characterized by multiple NNW-SSE trending fault-bounded ranges that expose thick $(>1 \mathrm{~km})$ Miocene volcanic sequences erupted both syn- and postsubduction (Fig. 1). There is a conspicuous absence of volcanism in all of southern Sonora between approximately 15 and $12.5 \mathrm{Ma}$. This period of volcanic quiescence is coincident with the cessation of subduction west of Baja based on plate reconstructions from geomagnetic data. A more thorough discussion of the geology, structure, geochronology and sedimentology of these regions of Sonora can be found in associated studies (Gans, 1997; Blair and Gans, 2003; MacMillan et al., 2003; Wong and Gans, 2003; Blair and Gans, 2005; Herman and Gans, 2005; MacMillan et al., 2005; Gans et al., 2006; Wong and Gans, 2008).

\section{Methods}

Because of the large geographic area covered by this geochemical survey, our approach was to focus on regions where key ages of volcanism were well represented, such as coastal Sonora, rather than to equally sample all volcanic units in an E-W transect across southern Sonora. Sampling is biased toward more mafic units within all regions, as these are less likely to be affected by significant crustal contamination and crystal fractionation and more apt to reflect their source composition. 108 major-oxide and 120 trace element analyses were measured using X-ray fluorescence (XRF) and inductively coupled plasma mass spectrometry (ICP-MS), respectively, at Washington State University (Table 1; Suppl. Table S1). The ${ }^{40} \mathrm{Ar} /$ ${ }^{39} \mathrm{Ar}$ geochronology was completed at University of California, Santa Barbara and electron microprobe analyses of phenocryst compositions were conducted on the Jeol 733 Superprobe at the Massachusetts Institute of Technology. 74 geochemical samples have been dated directly and the remaining 46 samples have well-constrained ages based on their stratigraphic position and correlation with dated sections (Table 1 ). These dates represent only a subset of the geochronology of these regions, as they bear directly on the geochemical interpretation. Geologic mapping and detailed geochronology of the individual regions within the study area will be presented elsewhere. The period of volcanic quiescence between 15 and $12.5 \mathrm{Ma}$ was punctuated by the eruption of two volumetrically insignificant lava flows in southern Sonora, a dacite in Sierra Mazatan at 14.21 Ma and a basalt in Sierra Libre at 13.0 Ma (Mora-Klepeis and McDowell, 2004; Gans et al., 2006). The dacite has a trace element signature characteristic of subduction-related volcanism, while the basalt does not. We therefore, chose $14 \mathrm{Ma}$ as the division between syn- and post-subduction samples for comparison of major and trace element compositions, although the entire period between 15 and $12.5 \mathrm{Ma}$ is thought by the authors to represent the timing of subduction cessation at the latitude of this study based on plate reconstructions.

\section{Geochemical results}

Whole rock compositions range from calc-alkaline basalt to rhyolite with no distinct changes after subduction ceased (Table 1; Fig. 2). Samples older than 14 Ma exhibit a more homogeneous range of compositions (49-67\% $\mathrm{SiO}_{2}$ ) in part due to our sampling bias toward more mafic volcanic rocks. Six andesite samples between 21 and $10 \mathrm{Ma}$ have characteristics of high $\mathrm{Mg} \#$ andesites $((\mathrm{Mg} / \mathrm{Mg}+\mathrm{Fe}) \times 100>0.45$ and 54-65 wt.\% $\mathrm{SiO}_{2}$ : Kelemen et al., 2003).

MORB-normalized trace element plots of all andesites (53 samples) and basalts (20 samples) exhibit a typical continental arc signature with the characteristic enrichment in LILE and depletion in HFSE. Both the andesites and basalts have persistent arc-like trace element signatures after subduction ceases (ca. $14 \mathrm{Ma}$ ). Detailed examination of these signatures reveals the subduction-related LILEenrichments, for example $\mathrm{Rb}$ and $\mathrm{Th}$ in the basalts, do decrease from $\sim 14$ Ma until volcanism ceased at $\sim 8$ Ma (Fig. 3; Suppl. Table S1). Similar to the change in LILE and HFSE, the rare earth elements (REE) patterns of the basalts become progressively less fractionated after subduction ceases (Fig. 4). These subtle changes in trace element behavior can be further quantified with trace element ratios. Ba/Ta is apt to reflect a fluid component from subducted oceanic crust and/or sediments due to the solubility of Ba in aqueous fluids relative to Ta (Spera et al., 2007). Ba/Ta values are similar during and after 


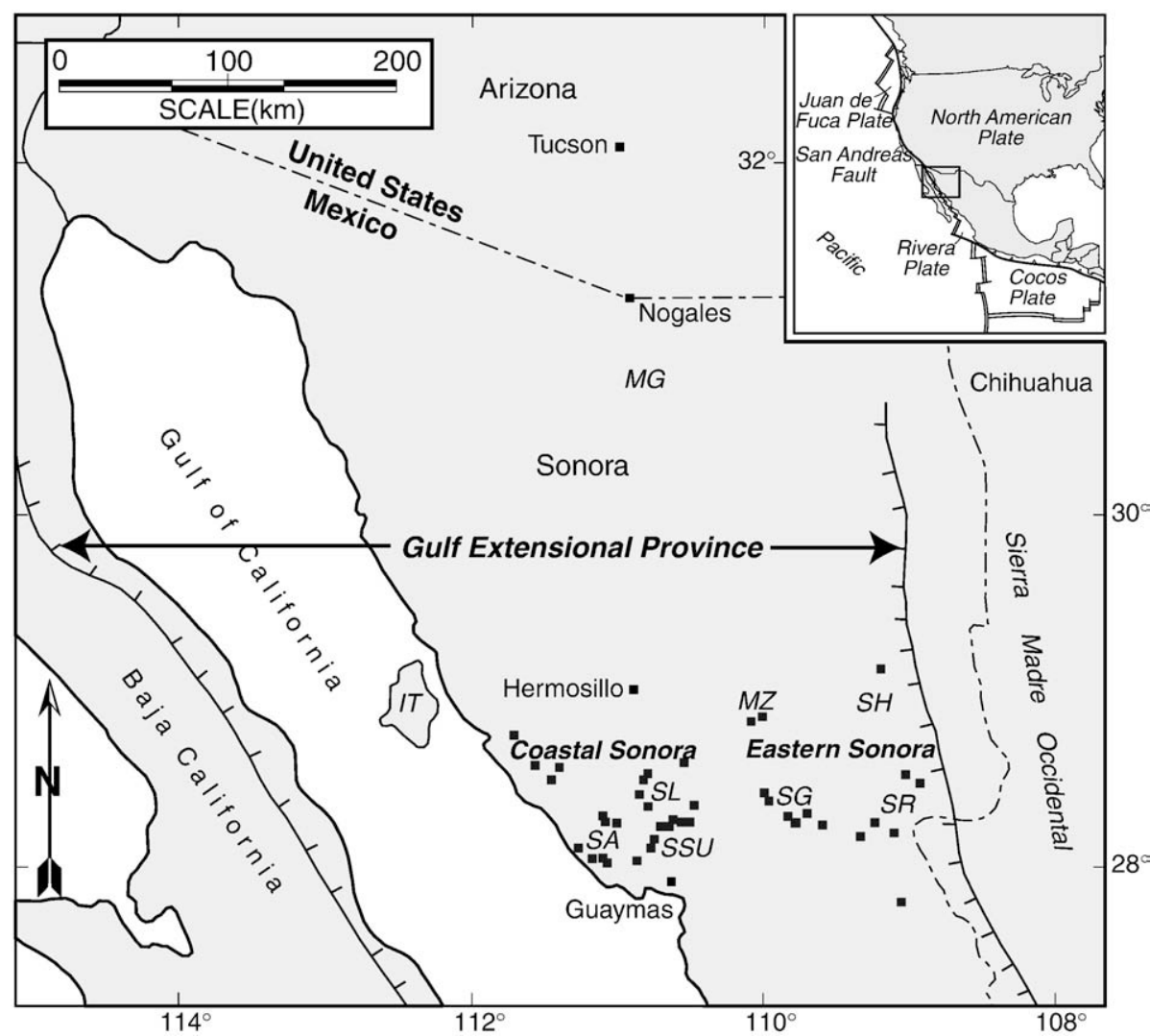

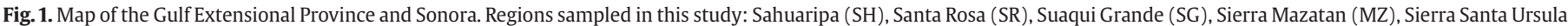

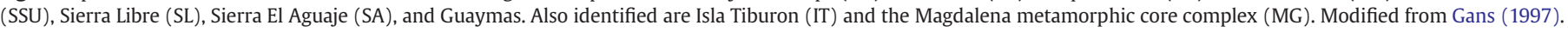

subduction, although the maximum Ba/Ta values decrease substantially after subduction ceases (Fig. 4). Dy/Yb ratios, which reflect the fractionation of the middle and heavy REE, are also similar before and after subduction but exhibit a decrease in maximum values after subduction ceased (Fig. 4).

An independent method to identify differences between syn- and post-subduction lavas is to evaluate their water content. We calculate the pre-eruptive $\mathrm{H}_{2} \mathrm{O}$-content of Sonoran lavas by simultaneous solution of the olivine-liquid geothermometer and olivine-clinopyroxene-plagioclase hygrometer of Sisson and Grove (1993). If synsubduction lavas are indeed the product of hydrous melting of the mantle wedge during subduction, as indicated by their continental arc trace element signatures, we expect them to have relatively high water contents ( $>4 \mathrm{wt} . \%$ ). If post-subduction lavas are the product of anhydrous decompression melting of asthenospheric mantle, then we expect them to be relatively water-poor. Alternately, if post-subduction lavas are the product of decompression melting or conductively reheating mantle lithosphere previously modified by subductionrelated fluid or melt, we might expect them to exhibit intermediate to low water contents (2-4 wt.\%) due to the breakdown of residual hydrous phases such as amphibole or phlogopite. Six mafic samples (Mg\#>0.5) ranging in age from 26.4 to 8.9 Ma multiply saturated with olivine, clinopyroxene, and plagioclase were first chosen as a sample set. The composition of the olivine phenocrysts were determined by electron microprobe and used in conjunction with the whole rock major and trace element analyses for thermometric and hygrometric calculations (Suppl. Table S2). For the calculations, we assume a pressure of $1 \mathrm{GPa}$ and that whole rock compositions approximate liquid compositions. We find a good correlation $\left(R^{2}=0.97\right)$ between the calculated pre-eruptive $\mathrm{H}_{2} \mathrm{O}$-content and temperature of crystallization. The six test samples range in pre-eruptive $\mathrm{H}_{2} \mathrm{O}$-content from 0.50 to 2.50 wt.\% $\mathrm{H}_{2} \mathrm{O}$ (Fig. 5). This method was then applied to the 71 most mafic samples from Sonora that range in age from 28.3 to $8.9 \mathrm{Ma}$ and for which the composition of the liquidus olivine was calculated assuming $K_{\mathrm{D}}^{\mathrm{Fe}-\mathrm{Mg}}=0.29$ as in Sisson and Grove (1993). These 71 samples also demonstrate a good correlation $\left(R^{2}=0.95\right)$ between the calculated pre-eruptive $\mathrm{H}_{2} \mathrm{O}$-content and temperature of crystallization. Samples erupted during subduction (28.3-15 Ma) vary from 0 to 7.9 wt.\% $\mathrm{H}_{2} \mathrm{O}$ (Fig. 5a). Samples erupted after 12.5 Ma show a decline from 5.4 to 0 wt.\% $\mathrm{H}_{2} \mathrm{O}$ over 3.5 m.y. When pre-eruptive $\mathrm{H}_{2} \mathrm{O}$ content is plotted against longitude, it is evident all the driest samples $\left(<2\right.$ wt.\% $\mathrm{H}_{2} \mathrm{O}$ ) erupted in the coastal region of Sonora (Fig. 5b).

\section{Discussion}

27-8 Ma mafic volcanic rocks erupted in southern Sonora overall have typical arc signatures but reveal subtle changes in their trace element concentrations after subduction ceases as demonstrated by their spider patterns and trace element ratios. There is a distinct decrease in the maximum value of chemical subduction indicators, such as $\mathrm{H}_{2} \mathrm{O}$-content, $\mathrm{Ba} / \mathrm{Ta}$, Dy/Yb, and $\mathrm{Rb}$-content, when subduction ceases for individual samples as well as the entire suite of mafic samples (Figs. 4a, b and 5a). The simplest interpretation of these observations and calculations is that the trace element abundances and $\mathrm{H}_{2} \mathrm{O}$-contents represent the abundance of metasomatized (slab fluid- or melt-modified) peridotite in the mantle at the time each melt was formed. Minimum Nb and Ta values are similar to average MORB values and therefore consistent with models attributing arc signatures to the preferential enrichment and depletion of the LILE and HFSE, respectively, in the mantle wedge by the addition of slab fluids or melts. Work on Mt. Shasta andesites by Grove et al. (2002) suggests LREE enrichments and $\mathrm{Dy} / \mathrm{Yb}$ concentrations in rocks similar to Sonoran syn-subduction andesites and basalts can be attributed to a subduction-related fluid-rich component in the melting region. Therefore the change over time in LREE, trace element ratios, and 
Table 1

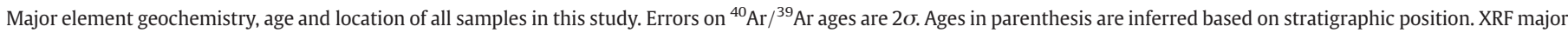
oxide chemistry is normalized to $100 \%$.

\begin{tabular}{|c|c|c|c|c|c|c|c|c|c|c|c|c|c|c|c|c|c|}
\hline Sample ID & $\mathrm{XRF}$ & ICPMS & $\mathrm{SiO} 2$ & $\mathrm{TiO} 2$ & $\mathrm{Al} 2 \mathrm{O} 3$ & $\mathrm{FeO}$ & $\mathrm{MnO}$ & $\mathrm{MgO}$ & $\mathrm{CaO}$ & $\mathrm{Na} 2 \mathrm{O}$ & $\mathrm{K} 2 \mathrm{O}$ & $\mathrm{P} 2 \mathrm{O} 5$ & Total & ${ }^{40} \mathrm{Ar} /{ }^{39} \mathrm{Ar}$ age & Reference & $\begin{array}{l}\text { Easting } \\
\text { (UTM) }\end{array}$ & $\begin{array}{l}\text { Northing } \\
\text { (UTM) }\end{array}$ \\
\hline \multicolumn{18}{|l|}{ Santa Rosa } \\
\hline $95 \mathrm{SN}-1$ & $*$ & $*$ & 60.13 & 0.955 & 17.15 & 6 & 0.106 & 2.81 & 6.15 & 3.79 & 2.51 & 0.403 & 99.61 & $17.45 \pm 0.10 \mathrm{Ma}$ & 1 & 670410 & 3148360 \\
\hline 95SR-11 & $*$ & $*$ & 54.57 & 1.309 & 17.73 & 8.34 & 0.138 & 3.72 & 8.09 & 3.53 & 1.94 & 0.628 & 99.19 & $26.40 \pm 0.50 \mathrm{Ma}$ & 1 & 680300 & 3157850 \\
\hline 95SR-56 & & $*$ & - & - & - & - & - & - & - & - & - & - & - & $22.60 \pm 0.30 \mathrm{Ma}$ & 1 & 680450 & 3159425 \\
\hline 95SR-72 & $*$ & * & 54.29 & 1.284 & 17.51 & 8.34 & 0.138 & 4.6 & 8.02 & 3.39 & 1.82 & 0.613 & 99.19 & $26.40 \pm 0.50 \mathrm{Ma}$ & 1 & 682675 & 3157650 \\
\hline 95SR-101 & $*$ & $*$ & 56.12 & 1.223 & 17.12 & 7.45 & 0.116 & 4.48 & 7.04 & 3.67 & 2.31 & 0.48 & 98.97 & $18.00 \pm 0.40 \mathrm{Ma}$ & 1 & 669890 & 3134325 \\
\hline \multicolumn{18}{|l|}{ Suaqui Grande } \\
\hline $96 \mathrm{SN}-010$ & $*$ & $*$ & 63.89 & 0.684 & 15.93 & 4.97 & 0.092 & 2.77 & 5.06 & 3.18 & 3.3 & 0.134 & 100 & $61.58 \pm 0.32 \mathrm{Ma}$ & 2 & 633680 & 3133840 \\
\hline 96SN-019 & $*$ & $*$ & 70.41 & 0.383 & 15.18 & 2.67 & 0.038 & 1.18 & 2.32 & 3.61 & 4.05 & 0.154 & 100 & $26.34 \pm 0.22 \mathrm{Ma}$ & 2 & 614050 & 3138599 \\
\hline $96 S N-32$ & & $*$ & - & - & - & - & - & - & - & - & - & - & - & $27.60 \pm 0.40 \mathrm{Ma}$ & 2 & 643460 & 3142759 \\
\hline $96 \mathrm{SN}-33$ & $*$ & * & 52.04 & 1.533 & 18.69 & 8.81 & 0.124 & 3.84 & 8.8 & 3.73 & 1.67 & 0.777 & 100 & $25.93 \pm 0.06 \mathrm{Ma}$ & 2 & 643410 & 3143838 \\
\hline $96 \mathrm{SN}-046$ & $*$ & $*$ & 67.12 & 0.498 & 15.61 & 3.9 & 0.061 & 1.88 & 4 & 3.35 & 3.47 & 0.119 & 100 & $59.27 \pm 0.30 \mathrm{Ma}$ & 2 & 633120 & 3157250 \\
\hline $96 \mathrm{SN}-060$ & $*$ & $*$ & 56.19 & 0.919 & 18.93 & 7.1 & 0.105 & 3.02 & 7.01 & 4.1 & 2.15 & 0.469 & 100 & $21.40 \pm 1.60 \mathrm{Ma}$ & 2 & 627070 & 3146997 \\
\hline $96 S N-73$ & & $*$ & - & - & - & - & - & - & - & - & - & - & - & $25.84 \pm 0.14 \mathrm{Ma}$ & 2 & 618430 & 3152598 \\
\hline $96 \mathrm{SN}-076$ & $*$ & $*$ & 78.55 & 0.117 & 11.53 & 1.51 & 0.041 & 0.31 & 1.73 & 2.11 & 4.08 & 0.017 & 100 & $12.41 \pm 0.06 \mathrm{Ma}$ & 2 & 617000 & 3126499 \\
\hline $96 \mathrm{SN}-077$ & $*$ & $*$ & 75.92 & 0.236 & 13.77 & 1.53 & 0.05 & 1.51 & 4 & 0.63 & 2.29 & 0.06 & 100 & $24.45 \pm 0.10 \mathrm{Ma}$ & 2 & 616260 & 3126818 \\
\hline $96 S N-82$ & & * & - & - & - & - & - & - & - & - & - & - & - & $25.81 \pm 0.12 \mathrm{Ma}$ & 2 & 612820 & 3143729 \\
\hline $96 \mathrm{SN}-088$ & $*$ & $*$ & 57.1 & 0.73 & 19.32 & 7.08 & 0.083 & 2.02 & 7 & 3.97 & 2.3 & 0.399 & 100 & $27.30 \pm 1.00 \mathrm{Ma}$ & 2 & 611990 & 3150460 \\
\hline $96 S N-106$ & $*$ & $*$ & 52.06 & 1.784 & 17.99 & 9.95 & 0.146 & 3.44 & 8.09 & 3.84 & 2 & 0.698 & 100.06 & $25.60 \pm 0.60 \mathrm{Ma}$ & 2 & 608340 & 3159640 \\
\hline $96 S N-131$ & & $*$ & - & - & - & - & - & - & - & - & - & - & - & $20.59 \pm 0.10 \mathrm{Ma}$ & 2 & 638620 & 3144360 \\
\hline $96 \mathrm{SN}-137$ & & * & - & - & - & - & - & - & - & - & - & - & - & $26.3 \pm 0.40 \mathrm{Ma}$ & 2 & 636610 & 3143700 \\
\hline $96 \mathrm{SN}-145$ & & * & - & - & - & - & - & - & - & - & - & - & - & $25.66 \pm 0.14 \mathrm{Ma}$ & 2 & 638900 & 3146160 \\
\hline $96 \mathrm{SN}-147$ & $*$ & * & 52.68 & 1.605 & 17.37 & 9.18 & 0.148 & 4.37 & 8.9 & 3.53 & 1.77 & 0.449 & 99.41 & $20.65 \pm 0.08 \mathrm{Ma}$ & 2 & 635950 & 3146200 \\
\hline \multicolumn{18}{|l|}{ Sahuaripa } \\
\hline 02KBS-013 & $*$ & * & 57.65 & 1.043 & 18.13 & 6.08 & 0.09 & 2.27 & 7.09 & 4.07 & 3.01 & 0.556 & 100 & $15.2 \pm 0.10 \mathrm{Ma}$ & 3 & 672268 & 3196317 \\
\hline 02KBS-017 & $*$ & $*$ & 51.76 & 2.136 & 16.92 & 10.43 & 0.148 & 4.33 & 7.95 & 3.77 & 1.69 & 0.878 & 100 & $24.87 \pm 0.10 \mathrm{Ma}$ & 3 & 673548 & 3219510 \\
\hline 02KBS-029 & $*$ & $*$ & 50.75 & 1.978 & 16.9 & 10.11 & 0.102 & 4.68 & 9.63 & 3.57 & 1.46 & 0.825 & 100 & $28.30 \pm 1.0 \mathrm{Ma}$ & 3 & 667798 & 3229433 \\
\hline 02KBS-030 & $*$ & $*$ & 61.36 & 0.757 & 17.82 & 4.9 & 0.096 & 2.61 & 5.77 & 3.91 & 2.5 & 0.276 & 100 & $16.16 \pm 0.18 \mathrm{Ma}$ & 3 & 673001 & 3189776 \\
\hline 02KBS-035 & $*$ & $*$ & 50.98 & 2.139 & 17.43 & 9.36 & 0.142 & 4.46 & 9.32 & 3.45 & 1.39 & 1.326 & 100 & $27.53 \pm 0.1 \mathrm{Ma}$ & 3 & 680651 & 3200177 \\
\hline 02KBS-038 & $*$ & $*$ & 62.48 & 0.711 & 17.4 & 4.65 & 0.094 & 2.2 & 5.72 & 3.79 & 2.74 & 0.219 & 100 & $15.92 \pm 0.06 \mathrm{Ma}$ & 3 & 676987 & 3187455 \\
\hline 02KBS-043 & $*$ & $*$ & 52.93 & 1.612 & 18.88 & 8.47 & 0.167 & 3.61 & 8.03 & 3.61 & 2.21 & 0.495 & 100 & $27.34 \pm 0.08 \mathrm{Ma}$ & 3 & 680652 & 3204841 \\
\hline 03KBS-57 & $*$ & * & 53.44 & 1.683 & 17.24 & 9.51 & 0.122 & 3.53 & 8.06 & 3.43 & 2.19 & 0.794 & 99.32 & $25.90 \pm 0.20 \mathrm{Ma}$ & 3 & 669719 & 3227601 \\
\hline 03KBS-059 & $*$ & $*$ & 50.92 & 2.326 & 16.85 & 9.54 & 0.209 & 4.71 & 8.83 & 3.66 & 1.64 & 1.32 & 100 & $27.68 \pm 0.10 \mathrm{Ma}$ & 3 & 673626 & 3216012 \\
\hline 03KBS-081 & $*$ & $*$ & 47.93 & 2.254 & 16.42 & 11.05 & 0.184 & 7.43 & 9.64 & 3.54 & 1.04 & 0.517 & 100 & $19.12 \pm 1.0 \mathrm{Ma}$ & 3 & 679823 & 3180368 \\
\hline \multicolumn{18}{|l|}{ Sierra Libre } \\
\hline 04CTSL-002 & $*$ & $*$ & 47.57 & 1.291 & 17.54 & 9.05 & 0.15 & 8.69 & 12.44 & 2.94 & 0.23 & 0.115 & 99.33 & $(\sim 13.0 \mathrm{Ma})$ & 4 & 508984 & 3171898 \\
\hline 02IMSL-01 & $*$ & $*$ & 75.44 & 0.147 & 12.53 & 1.93 & 0.027 & 0.03 & 0.22 & 3.35 & 6.32 & 0.02 & 99.32 & $12.34 \pm 0.04 \mathrm{Ma}$ & 4 & 497280 & 3151172 \\
\hline 02IMSL-02 & $*$ & * & 61.81 & 0.746 & 16.98 & 5.25 & 0.065 & 2.78 & 5.73 & 3.88 & 2.54 & 0.225 & 98.9 & $(\sim 11.9 \mathrm{Ma})$ & 4 & 495805 & 3150393 \\
\hline 02IMSL-03 & $*$ & $*$ & 51.45 & 1.567 & 16.83 & 10.61 & 0.149 & 5.7 & 9.42 & 3.25 & 0.87 & 0.157 & 98.34 & $13.0 \pm 0.3 \mathrm{Ma}$ & 4 & 496106 & 3150481 \\
\hline 02IMSL-04 & $*$ & $*$ & 59.15 & 1.913 & 14.98 & 8.72 & 0.163 & 2.44 & 5.25 & 4.42 & 2.47 & 0.49 & 99.52 & $(12.2-11.1 \mathrm{Ma})$ & 4 & 496221 & 3150460 \\
\hline H-IMSL-6A & $*$ & $*$ & 65.47 & 1.178 & 14.82 & 5.92 & 0.131 & 1.06 & 3.33 & 4.3 & 3.45 & 0.343 & 99 & (12.2-11.1 Ma) & 4 & & \\
\hline 02IMSL-09 & $*$ & $*$ & 75.62 & 0.148 & 12.44 & 2 & 0.034 & 0 & 0.55 & 3.53 & 5.67 & 0.011 & 97.49 & (12.2-11.1 Ma) & 4 & 499617 & 3150499 \\
\hline H-IMSL-11 & $*$ & $*$ & 67.55 & 0.482 & 14.09 & 6.66 & 0.164 & 0.01 & 0.88 & 5.17 & 4.93 & 0.074 & 97.96 & (12.2-11.1 Ma) & 4 & & \\
\hline H-IMSL-12 & $*$ & $*$ & 75.61 & 0.172 & 12.25 & 2.19 & 0.047 & 0 & 0.7 & 3.43 & 5.59 & 0.013 & 97.02 & $(12.2-11.1 \mathrm{Ma})$ & 4 & $528720^{\circ}$ & 3145447 \\
\hline 02IMSL-13 & $*$ & $*$ & 60.9 & 1.322 & 14.77 & 7.18 & 0.127 & 3.25 & 5.91 & 3.41 & 2.86 & 0.271 & 99.4 & $10.26 \pm 0.04 \mathrm{Ma}$ & 4 & 511297 & 3141602 \\
\hline 02IMSL-15 & $*$ & $*$ & 76.7 & 0.154 & 11.88 & 1.95 & 0.036 & 0 & 0.59 & 3.41 & 5.27 & 0.012 & 97.19 & $12.15 \pm 0.04 \mathrm{Ma}$ & 4 & 528720 & 3145447 \\
\hline 02IMSL-19 & $*$ & $*$ & 58.65 & 1.35 & 16.78 & 6.63 & 0.109 & 3.92 & 6.85 & 4.05 & 1.34 & 0.329 & 99.46 & $(10.7-10.2 \mathrm{Ma})$ & 4 & 492096 & 3146456 \\
\hline H-IMSL-19a & $*$ & $*$ & 75.31 & 0.121 & 12.78 & 1.86 & 0.058 & 0.06 & 0.92 & 3.77 & 5.09 & 0.021 & 98.97 & $(12.2-11.1 \mathrm{Ma})$ & 4 & & \\
\hline H-IMSL-19b & $*$ & $*$ & 75.04 & 0.134 & 13.07 & 1.95 & 0.026 & 0.03 & 0.9 & 3.75 & 5.06 & 0.057 & 98.56 & (12.2-11.1 Ma) & 4 & & \\
\hline 02IMSL-22 & $*$ & $*$ & 68.18 & 0.45 & 14.06 & 6.3 & 0.166 & 0.02 & 0.79 & 4.94 & 5.04 & 0.054 & 98.46 & (12.2-11.1 Ma) & 4 & 497867 & 3148997 \\
\hline H-IMSL-24 & $*$ & $*$ & 69.35 & 0.791 & 13.65 & 4.17 & 0.102 & 0.87 & 2.53 & 4.14 & 4.21 & 0.196 & 98.69 & $(12.2-11.1 \mathrm{Ma})$ & 4 & & \\
\hline H-IMSL-24a & $*$ & $*$ & 62.89 & 1.514 & 14.9 & 7.39 & 0.124 & 1.7 & 4 & 4.18 & 2.94 & 0.383 & 98.57 & (12.2-11.1 Ma) & 4 & & \\
\hline H-IMSL-24b & $*$ & $*$ & 58.98 & 1.905 & 15.14 & 8.55 & 0.161 & 2.39 & 5.23 & 4.67 & 2.46 & 0.496 & 99.34 & (12.2-11.1 Ma) & 4 & & \\
\hline H-IMSL-25 & $*$ & $*$ & 64.42 & 1.254 & 14.72 & 6.53 & 0.102 & 1.43 & 3.7 & 4.25 & 3.23 & 0.363 & 99.01 & (12.2-11.1 Ma) & 4 & & \\
\hline H-IMSL-28 & $*$ & $*$ & 75.86 & 0.147 & 12.41 & 2.05 & 0.038 & 0 & 0.59 & 3.98 & 4.92 & 0.014 & 97.3 & (12.2-11.1 Ma) & 4 & 497932 & 3152649 \\
\hline 03IMSL-29 & $*$ & $*$ & 66.44 & 0.532 & 16.06 & 3.73 & 0.07 & 1.87 & 3.88 & 3.96 & 3.24 & 0.21 & 98.29 & $19.5 \pm 0.1 \mathrm{Ma}$ & 4 & 496600 & 3153832 \\
\hline H-IMSL-31 & $*$ & $*$ & 64.37 & 1.256 & 14.78 & 6.51 & 0.143 & 1.31 & 3.76 & 4.24 & 3.26 & 0.372 & 98.84 & $(12.2-11.1 \mathrm{Ma})$ & 4 & 526832 & 3140499 \\
\hline 04IMSL-31 & $*$ & $*$ & 61.41 & 0.741 & 17.41 & 5.01 & 0.078 & 2.22 & 5.83 & 3.89 & 3.08 & 0.323 & 98.77 & (12.2-11.1 Ma) & 4 & 526832 & 3140499 \\
\hline 04IMSL-39 & $*$ & $*$ & 76.28 & 0.134 & 12.62 & 1.95 & 0.043 & 0.07 & 0.83 & 4.69 & 3.37 & 0.013 & 96.77 & $12.34 \pm 0.04 \mathrm{Ma}$ & 4 & 517461 & 3165400 \\
\hline \multicolumn{18}{|c|}{ Sierra Santa Ursula } \\
\hline 04CTSSU-002 & $*$ & $*$ & 57.84 & 0.841 & 17.04 & 6.31 & 0.108 & 4.06 & 6.8 & 3.8 & 2.77 & 0.422 & 97.9 & $(\sim 23 \mathrm{Ma})$ & 4 & 508737.3143 & 3119582.686 \\
\hline 04CTSSU-004 & $*$ & $*$ & 56 & 0.874 & 17.77 & 7.15 & 0.122 & 4.73 & 7.1 & 3.81 & 2.12 & 0.323 & 97.67 & $(\sim 23 \mathrm{Ma})$ & 4 & 509000.1714 & 3120139.943 \\
\hline 04CTSSU-005 & $*$ & $*$ & 49.27 & 1.535 & 12.94 & 7.99 & 0.14 & 11.47 & 9.85 & 3.48 & 2.23 & 1.101 & 97.87 & (21.3 Ma) & 4 & 509126.3429 & 3120728.743 \\
\hline 04CTSSU-006 & $*$ & $*$ & 63.9 & 0.66 & 16.79 & 4.46 & 0.071 & 1.57 & 5.19 & 4.13 & 2.93 & 0.294 & 98.98 & $(\sim 23 \mathrm{Ma})$ & 4 & 509904.4 & 3120423.829 \\
\hline 04CTSSU-007 & $*$ & $*$ & 54.16 & 0.77 & 16.83 & 7.38 & 0.128 & 7.29 & 8.66 & 3.46 & 1.15 & 0.167 & 99.26 & $(\sim 20.7 \mathrm{Ma})$ & 4 & 509862.3429 & 3119656.286 \\
\hline 04CTSSU-008 & $*$ & $*$ & 48.95 & 1.012 & 14.42 & 8.47 & 0.145 & 11.93 & 9.63 & 2.88 & 2.08 & 0.499 & 98.39 & $(\sim 21.3 \mathrm{Ma})$ & 4 & 509452.2857 & 3120318.686 \\
\hline 04CTSSU-009 & $*$ & $*$ & 70.86 & 0.407 & 14.54 & 3.26 & 0.069 & 0.41 & 2.24 & 3.86 & 4.26 & 0.094 & 99.16 & $(\sim 11.9 \mathrm{Ma})$ & 4 & 511176.6286 & 3119835.029 \\
\hline 04CTSSU-012 & $*$ & $*$ & 56.77 & 0.896 & 16.59 & 7.02 & 0.125 & 4.37 & 7.73 & 3.13 & 3.01 & 0.354 & 99.19 & $(\sim 21.6 \mathrm{Ma})$ & 4 & 509010.6857 & 3122579.257 \\
\hline 04CTSSU-013 & $*$ & * & 64.03 & 1.191 & 14.72 & 7.35 & 0.113 & 1.03 & 3.01 & 4.1 & 4.04 & 0.424 & 99.04 & $(\sim 12 \mathrm{Ma})$ & 4 & 513069.2 & 3119656.286 \\
\hline
\end{tabular}


Table 1 (continued)

\begin{tabular}{|c|c|c|c|c|c|c|c|c|c|c|c|c|c|c|c|c|c|}
\hline Sample ID & XRF & ICPMS & $\mathrm{SiO} 2$ & $\mathrm{TiO} 2$ & $\mathrm{Al} 2 \mathrm{O3}$ & $\mathrm{FeO}$ & $\mathrm{MnO}$ & $\mathrm{MgO}$ & $\mathrm{CaO}$ & $\mathrm{Na} 2 \mathrm{O}$ & $\mathrm{K} 2 \mathrm{O}$ & $\mathrm{P} 2 \mathrm{O} 5$ & Total & ${ }^{40} \mathrm{Ar} /{ }^{39} \mathrm{Ar}$ age & Reference & $\begin{array}{l}\text { Easting } \\
\text { (UTM) }\end{array}$ & $\begin{array}{l}\text { Northing } \\
\text { (UTM) }\end{array}$ \\
\hline \multicolumn{18}{|c|}{$\begin{array}{l}\text { Sierra Santa Ursula } \\
\text { (cont.) }\end{array}$} \\
\hline 04CTSSU-015 & $*$ & $*$ & 55.92 & 2.538 & 13.53 & 11.13 & 0.168 & 3 & 7.5 & 3.35 & 2.38 & 0.486 & 97.98 & $(\sim 11.7 \mathrm{Ma})$ & 4 & 514303.4642 & 3121710.179 \\
\hline 04CTSSU-016 & $*$ & $*$ & 52.3 & 1.649 & 16.57 & 9.21 & 0.153 & 6.02 & 9.25 & 3.36 & 1.15 & 0.327 & 99.38 & ( 20.7 Ma) & 4 & 517128.1786 & 3124631.464 \\
\hline 04IMSU-10 & $*$ & $*$ & 56.43 & 0.858 & 17.6 & 6.79 & 0.108 & 4.34 & 7.41 & 3.5 & 2.66 & 0.304 & 98.49 & $21.6 \pm 0.4 \mathrm{Ma}$ & 4 & 507890 & 3121109 \\
\hline 04IMSU-11 & $*$ & $*$ & 54.72 & 0.939 & 16.24 & 7.19 & 0.125 & 7.63 & 7.92 & 3.4 & 1.54 & 0.288 & 99.63 & ( 20.7 Ma) & 4 & 507074 & 3123310 \\
\hline 04IMSU-13 & $*$ & $*$ & 58.87 & 0.807 & 17.97 & 6.15 & 0.064 & 2.83 & 6.95 & 3.64 & 2.4 & 0.309 & 98.62 & $(\sim 21.6 \mathrm{Ma})$ & 4 & 509141 & 3119528 \\
\hline 04IMSU-20 & $*$ & $*$ & 58.94 & 0.886 & 17.29 & 5.97 & 0.097 & 2.83 & 6.8 & 4.24 & 2.51 & 0.422 & 98.17 & $(23 \mathrm{Ma})$ & 4 & 511990 & 3122186 \\
\hline 04IMSU-23 & $*$ & $*$ & 55.89 & 1.078 & 17.39 & 7.15 & 0.124 & 5.1 & 7.06 & 3.86 & 1.97 & 0.38 & 98.83 & ( 20.7 Ma) & 4 & 512732 & 3119985 \\
\hline 04IMSU-24 & $*$ & $*$ & 62.81 & 1.352 & 14.72 & 7.88 & 0.132 & 1.23 & 3.52 & 4.09 & 3.74 & 0.529 & 98.88 & $(\sim 12 \mathrm{Ma})$ & 4 & 512866 & 3119975 \\
\hline 04IMSU-27 & $*$ & $*$ & 56.77 & 2.147 & 16.76 & 10.43 & 0.152 & 1.97 & 5.87 & 4.01 & 1.39 & 0.499 & 98.16 & ( 20.7 Ma) & 4 & 519517 & 3123420 \\
\hline \multicolumn{18}{|l|}{ Sierra El Aguaje } \\
\hline 02PGS-24 & $*$ & $*$ & 57.46 & 1.088 & 16.23 & 6.42 & 0.127 & 5.61 & 7.82 & 3.48 & 1.41 & 0.356 & 99.96 & $8.90 \pm 0.20 \mathrm{Ma}$ & 2 & 482825 & 3130739 \\
\hline 02PGS-25 & $*$ & $*$ & 65.92 & 0.661 & 16.66 & 3.64 & 0.07 & 2.06 & 4.81 & 4.45 & 1.55 & 0.187 & 100 & $9.00 \pm 0.22 \mathrm{Ma}$ & 2 & 481048 & 3131284 \\
\hline 02PGS-26 & $*$ & $*$ & 55.11 & 1.355 & 16.87 & 6.83 & 0.123 & 5.17 & 8.93 & 3.69 & 1.51 & 0.411 & 99.7 & $9.02 \pm 0.06 \mathrm{Ma}$ & 2 & 485462 & 3131156 \\
\hline 02PGS-28 & $*$ & $*$ & 51.21 & 2.12 & 17.19 & 8.94 & 0.154 & 5.82 & 8.75 & 4 & 1.25 & 0.563 & 100 & $9.12 \pm 0.06 \mathrm{Ma}$ & 2 & 493655 & 3110851 \\
\hline 02PGS-34 & $*$ & $*$ & 54.67 & 0.948 & 16.83 & 6.56 & 0.108 & 7.86 & 7.69 & 3.18 & 1.83 & 0.32 & 100.08 & $16.50 \pm 0.08 \mathrm{Ma}$ & 2 & 496410 & 3096776 \\
\hline 02PGS-35 & $*$ & $*$ & 54.35 & 2.063 & 16.39 & 9.23 & 0.162 & 4.06 & 7.64 & 3.69 & 1.99 & 0.426 & 98.96 & $10.80 \pm 0.20 \mathrm{Ma}$ & 2 & 496824 & 3096774 \\
\hline 02PGS-41 & $*$ & $*$ & 64.46 & 0.621 & 17.08 & 4.02 & 0.081 & 1.96 & 5.17 & 3.94 & 2.39 & 0.271 & 98.51 & $15.30 \pm 0.12 \mathrm{Ma}$ & 2 & 488910 & 3095400 \\
\hline 02PGS-42A & $*$ & $*$ & 77.99 & 0.052 & 12.27 & 0.78 & 0.032 & 0 & 0.44 & 3.83 & 4.6 & 0.023 & 99.91 & $10.70 \pm 0.16 \mathrm{Ma}$ & 2 & 487945 & 3100026 \\
\hline 03PGS-53B & $*$ & $*$ & 76.88 & 0.156 & 12.52 & 1.05 & 0.021 & 0.13 & 0.97 & 3.58 & 4.66 & 0.026 & 100 & $10.15 \pm 0.06 \mathrm{Ma}$ & 2 & 496173 & 3110007 \\
\hline 03PGS-54 & & $*$ & - & - & - & - & - & - & - & - & - & - & - & $10.13 \pm 0.05 \mathrm{Ma}$ & 2 & 496200 & 3110134 \\
\hline 03PGS-58 & $*$ & $*$ & 50.93 & 2.073 & 16.08 & 9.34 & 0.152 & 7.29 & 8.4 & 3.69 & 1.47 & 0.568 & 100 & $9.01 \pm 0.04 \mathrm{Ma}$ & 2 & 495160 & 3109337 \\
\hline 03PGS-62 & $*$ & $*$ & 64.07 & 0.614 & 16.73 & 4.89 & 0.099 & 2.51 & 5.15 & 3.33 & 2.47 & 0.143 & 100 & $10.66 \pm 0.22 \mathrm{Ma}$ & 2 & 482058 & 3098938 \\
\hline 03PGS-64 & $*$ & $*$ & 59.09 & 0.742 & 15.62 & 5.64 & 0.086 & 6.2 & 7.19 & 3.09 & 2.04 & 0.305 & 100.36 & $21.00 \pm 0.15 \mathrm{Ma}$ & 2 & 485825 & 3100435 \\
\hline 03PGS-67 & $*$ & $*$ & 67.33 & 0.591 & 16.21 & 3.27 & 0.061 & 1.65 & 3.91 & 4.06 & 2.75 & 0.168 & 100 & $8.32 \pm 0.15 \mathrm{Ma}$ & 2 & 482584 & 3109368 \\
\hline 03PGS-73 & $*$ & $*$ & 63.61 & 0.942 & 16.74 & 4.68 & 0.087 & 2.04 & 4.93 & 4.65 & 2.09 & 0.235 & 100 & $9.19+0.04 \mathrm{Ma}$ & 2 & 481824 & 3107627 \\
\hline 03PGS-74 & & $*$ & - & - & - & - & - & - & - & - & - & - & - & $9.91 \pm 0.06 \mathrm{Ma}$ & 2 & 482017 & 3104443 \\
\hline 03PGS-75 & $*$ & $*$ & 50.98 & 1.905 & 17.13 & 10.05 & 0.138 & 5.73 & 9.26 & 3.65 & 0.87 & 0.289 & 100 & $10.00 \pm 0.30 \mathrm{Ma}$ & 2 & 481391 & 3104783 \\
\hline 04PGS-081 & $*$ & $*$ & 56.76 & 1.189 & 17.05 & 6.67 & 0.095 & 4.3 & 6.98 & 3.64 & 2.83 & 0.474 & 100 & $11.50 \pm 0.15 \mathrm{Ma}$ & 2 & 491153 & 3100612 \\
\hline 04PGS-094 & $*$ & $*$ & 60.06 & 1.079 & 17.49 & 6.36 & 0.112 & 2.67 & 5.87 & 3.97 & 2.18 & 0.207 & 100 & $(<12.10 \mathrm{Ma})$ & 2 & 500341 & 3098786 \\
\hline 04PGS-104 & $*$ & $*$ & 70.1 & 0.763 & 14.07 & 4.05 & 0.066 & 0.57 & 1.74 & 3.74 & 4.72 & 0.181 & 100 & $(\sim 11.2-12 \mathrm{Ma})$ & 2 & 552039 & 3116903 \\
\hline 04CTSA-1 & $*$ & $*$ & 53.79 & 1.25 & 16.91 & 7.88 & 0.127 & 6.05 & 7.95 & 3.54 & 2.07 & 0.432 & 98.65 & (20 or $16 \mathrm{Ma})$ & 2 & 489521 & 3097494 \\
\hline O4CTSA-2 & $*$ & $*$ & 53.9 & 1.256 & 17.14 & 7.92 & 0.111 & 5.76 & 8.02 & 3.52 & 1.95 & 0.421 & 98.67 & (20 or $16 \mathrm{Ma})$ & 2 & 489552 & 3097509 \\
\hline 04CTSA-4 & $*$ & $*$ & 56.62 & 0.858 & 17.28 & 7.31 & 0.164 & 4.61 & 7.16 & 3.38 & 2.23 & 0.39 & 98.88 & $(\sim 15.7 \mathrm{Ma})$ & 2 & 489664 & 3097550 \\
\hline 04CTSA-5 & $*$ & $*$ & 58.42 & 0.884 & 16.51 & 6.35 & 0.104 & 5.3 & 6.65 & 3.48 & 1.97 & 0.335 & 98.23 & $(\sim 15.7 \mathrm{Ma})$ & 2 & 489725 & 3097624 \\
\hline 04CTSA-7 & $*$ & $*$ & 52.05 & 1.744 & 17.37 & 9.79 & 0.155 & 4.82 & 8.34 & 3.69 & 1.68 & 0.355 & 98.31 & $(\sim 11.9-11.3 \mathrm{Ma})$ & 2 & 489711 & 3097799 \\
\hline 04CTSA-8 & $*$ & $*$ & 55.65 & 1.813 & 16.31 & 9.09 & 0.132 & 3.45 & 6.63 & 4.05 & 2.44 & 0.433 & 99.08 & $(\sim 11.9-11.3 \mathrm{Ma})$ & 2 & 489711 & 3097799 \\
\hline 04CTSA-9 & $*$ & $*$ & 51.36 & 1.652 & 17.93 & 9.14 & 0.096 & 5.5 & 9.22 & 3.57 & 1.19 & 0.343 & 98.09 & $(\sim 11.9-11.3 \mathrm{Ma})$ & 2 & 489753 & 3097914 \\
\hline 04CTSA-10 & $*$ & $*$ & 51.13 & 2.039 & 17.14 & 9.97 & 0.156 & 5.26 & 8.75 & 3.71 & 1.44 & 0.393 & 98.05 & ( 11.9-11.3 Ма) & 2 & 489753 & 3097914 \\
\hline \multicolumn{18}{|l|}{ SE Sonora } \\
\hline 03PGS-Nuri1 & * & $*$ & 54.72 & 1.451 & 17.8 & 7.71 & 0.13 & 3.96 & 6.45 & 4.24 & 2.83 & 0.713 & 100.01 & $16.40 \pm 0.10 \mathrm{Ma}$ & 2 & 664591 & 3109940 \\
\hline \multicolumn{18}{|l|}{ Coastal Sonora } \\
\hline 01PGS-35A & $*$ & $*$ & 76.28 & 0.131 & 12.79 & 1.68 & 0.041 & 0.13 & 0.97 & 4.99 & 2.98 & 0.018 & 100 & $12.37 \pm 0.04 \mathrm{Ma}$ & 2 & 402169 & 3195182 \\
\hline 02PGS-01 & $*$ & $*$ & 76.1 & 0.124 & 12.63 & 1.62 & 0.04 & 0.09 & 0.68 & 4.13 & 4.57 & 0.015 & 100 & $12.25 \pm 0.10 \mathrm{Ma}$ & 2 & 500758 & 3203873 \\
\hline 02PGS-07 & $*$ & $*$ & 55.09 & 1.585 & 16.32 & 7.61 & 0.136 & 5.53 & 8.45 & 3.62 & 1.31 & 0.342 & 99.62 & $10.02 \pm 0.17 \mathrm{Ma}$ & 2 & 478909 & 3176173 \\
\hline 02PGS-8 & & $*$ & - & - & - & - & - & - & - & - & - & - & - & $10.70 \pm 0.25 \mathrm{Ma}$ & 2 & 478706 & 3176107 \\
\hline 02PGS-09 & $*$ & $*$ & 54.37 & 1.347 & 16.36 & 7.21 & 0.127 & 6.39 & 9.23 & 3.48 & 1.15 & 0.33 & 99.32 & $10.65 \pm 0.06 \mathrm{Ma}$ & 2 & 476427 & 3169507 \\
\hline 02PGS-12 & $*$ & $*$ & 63.04 & 1.318 & 15.64 & 5.57 & 0.108 & 1.98 & 4.74 & 4.05 & 3.21 & 0.342 & 100 & $11.41 \pm 0.04 \mathrm{Ma}$ & 2 & 471891 & 3163132 \\
\hline 02PGS-13 & $*$ & $*$ & 50.54 & 1.537 & 16.88 & 8.53 & 0.162 & 7.51 & 10.8 & 3.33 & 0.5 & 0.21 & 100 & $9.50 \pm 0.15 \mathrm{Ma}$ & 2 & 47563 & 3155326 \\
\hline 02PGS-15 & $*$ & $*$ & 51.01 & 1.575 & 16.85 & 8.61 & 0.161 & 7.37 & 10.28 & 3.31 & 0.61 & 0.237 & 100 & $9.00 \pm 1.00 \mathrm{Ma}$ & 2 & 471831 & 3154577 \\
\hline 02PGS-19 & & $*$ & - & - & - & - & - & - & - & - & - & - & - & $8.25 \pm 0.25 \mathrm{Ma}$ & 2 & 459283 & 3133526 \\
\hline \multicolumn{18}{|l|}{ Guaymas } \\
\hline 02PGS-32 & $*$ & $*$ & 56.27 & 0.787 & 16.92 & 6.79 & 0.118 & 7.09 & 7.08 & 3.51 & 1.26 & 0.169 & 100.1 & $17.00 \pm 0.50 \mathrm{Ma}$ & 2 & 500544 & 3100191 \\
\hline 03PGS-50 & $*$ & $*$ & 49.91 & 2.005 & 16.19 & 10.33 & 0.179 & 7.41 & 9.69 & 3.27 & 0.65 & 0.366 & 99.05 & $10.40 \pm 0.10 \mathrm{Ma}$ & 2 & 531557 & 3091086 \\
\hline \multicolumn{18}{|l|}{ Sierra Mazatan } \\
\hline 01PGS-02 & $*$ & $*$ & 61.08 & 0.762 & 17.18 & 5.24 & 0.092 & 2.86 & 6.03 & 3.83 & 2.65 & 0.274 & 100 & $24.20 \pm 0.10$ & 5 & 548471 & 3213558 \\
\hline 02MAZ-069 & $*$ & $*$ & 76.31 & 0.12 & 12.6 & 1.56 & 0.017 & 0.16 & 0.33 & 3.69 & 5.16 & 0.062 & 100 & $12.44 \pm 0.10 \mathrm{Ma}$ & 5 & 581811 & 3233061 \\
\hline 03MAZ-83 & $*$ & $*$ & 64.08 & 0.671 & 16.71 & 4.08 & 0.089 & 2.25 & 5.33 & 3.88 & 2.68 & 0.23 & 100 & $14.21 \pm 0.06 \mathrm{Ma}$ & 5 & 569665 & 3235475 \\
\hline 03MAZ-129 & $*$ & $*$ & 56.18 & 1.411 & 18.86 & 3.81 & 0.14 & 1.52 & 8.73 & 4.58 & 3.78 & 1 & 100 & $16.50 \pm 0.50 \mathrm{Ma}$ & 5 & 574930 & 3235209 \\
\hline 03MAZ-132 & $*$ & $*$ & 58.04 & 1.17 & 17.64 & 6.3 & 0.131 & 1.71 & 6.34 & 4.38 & 3.51 & 0.785 & 100 & $17.86 \pm 0.06 \mathrm{Ma}$ & 5 & 572770 & 3241435 \\
\hline 03MAZ-078 & $*$ & $*$ & 62.49 & 0.759 & 17.08 & 4.82 & 0.087 & 2.4 & 5.48 & 3.83 & 2.78 & 0.28 & 100 & $15.19 \pm 0.12 \mathrm{Ma}$ & 5 & 581180 & 3236375 \\
\hline
\end{tabular}

References:

$1=$ Gans, 1997.

$2=$ Gans et al., 2006 and Gans, pers. comm., 2009.

3=Blair and Gans, 2003, 2005 and Blair, pers. comm., 2006.

4=MacMillan et al., 2003, 2005 and MacMillan, pers. comm., 2006.

5=Wong and Gans, 2003, 2008. 


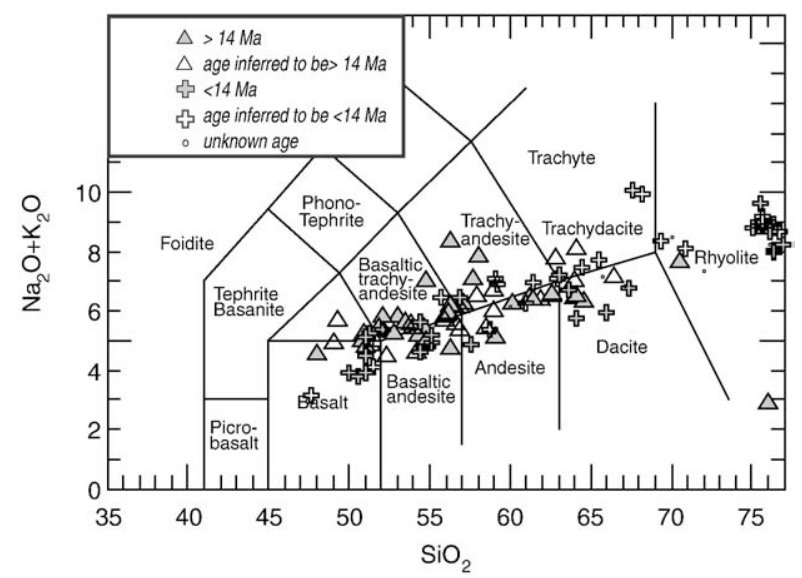

Fig. 2. Alkali-silica classification diagram (Le Bas et al., 1992) for all samples divided by age.
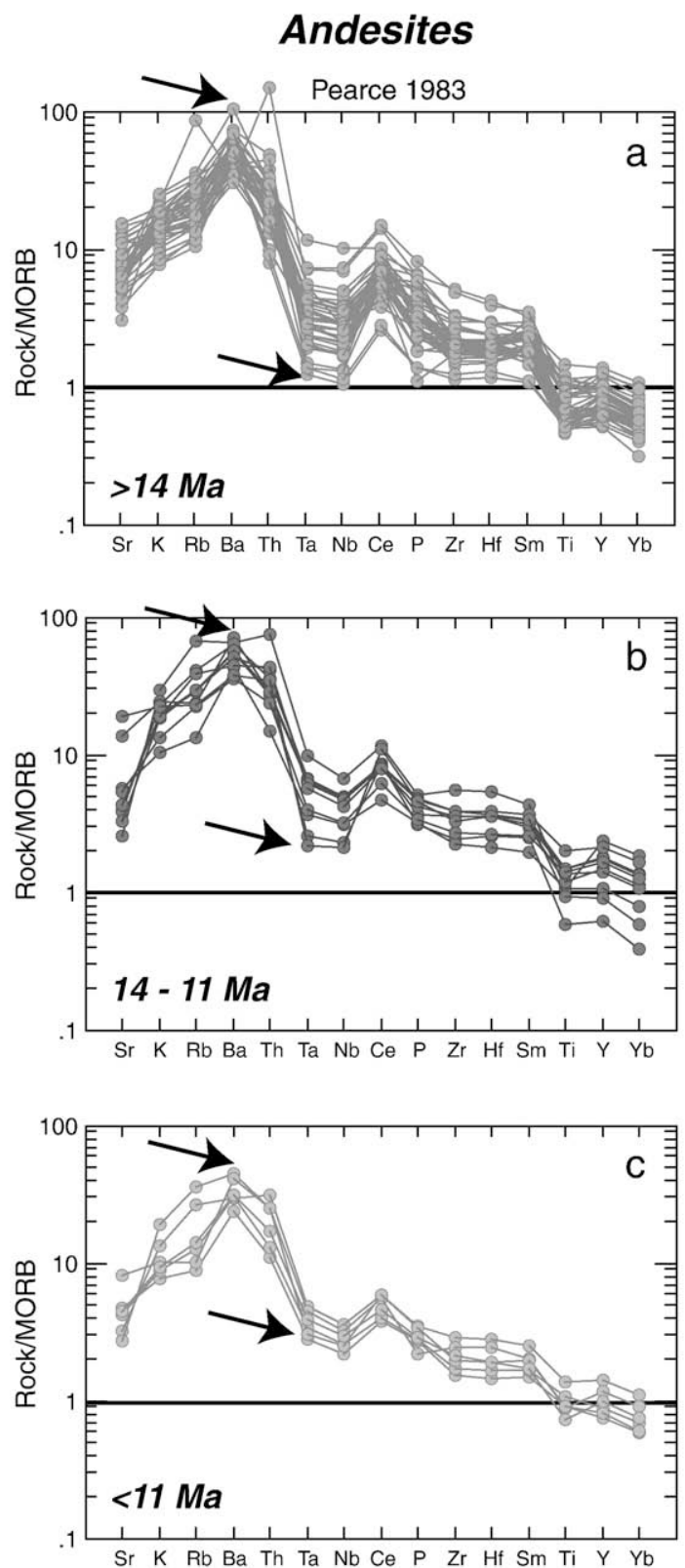

$\mathrm{H}_{2} \mathrm{O}$-contents is consistent with a decrease in the amount of metasomatized mantle in the source region once subduction ceases.

The residual arc characteristics in $<14$ Ma volcanism suggests the time required to completely erase the subduction signature from the sub-arc mantle was greater than the 4 million years of volcanism that post-dated subduction in Sonora. Intermediate $\mathrm{H}_{2} \mathrm{O}$-content (2-4 wt.\%) post-subduction magmas likely formed when crustal extension in Sonora trigged conductive reheating or decompression melting of reservoirs of mantle lithosphere previously modified by subductionrelated fluid or melt (Fig. 6). The high $\mathrm{Mg}$ \# andesites erupted during subduction have arc trace element signatures and likely represent water-rich primary melts of the mantle wedge rather than melts of oceanic slab (Grove et al., 2002). Post-subduction high Mg\# andesites were likely formed by the remobilization of lithosphere reservoirs of water-rich primary melts. Coastal Sonora was the focus of $<12.5 \mathrm{Ma}$ proto-Gulf extension and consequent melting, which likely explains the range of post-subduction $\mathrm{H}_{2} \mathrm{O}$-contents found here from intermediate $\mathrm{H}_{2} \mathrm{O}$-contents (2-4 wt.\%) to the minimum values observed ( $\left.<1 \mathrm{wt} . \%\right)$.

\section{Basalts}
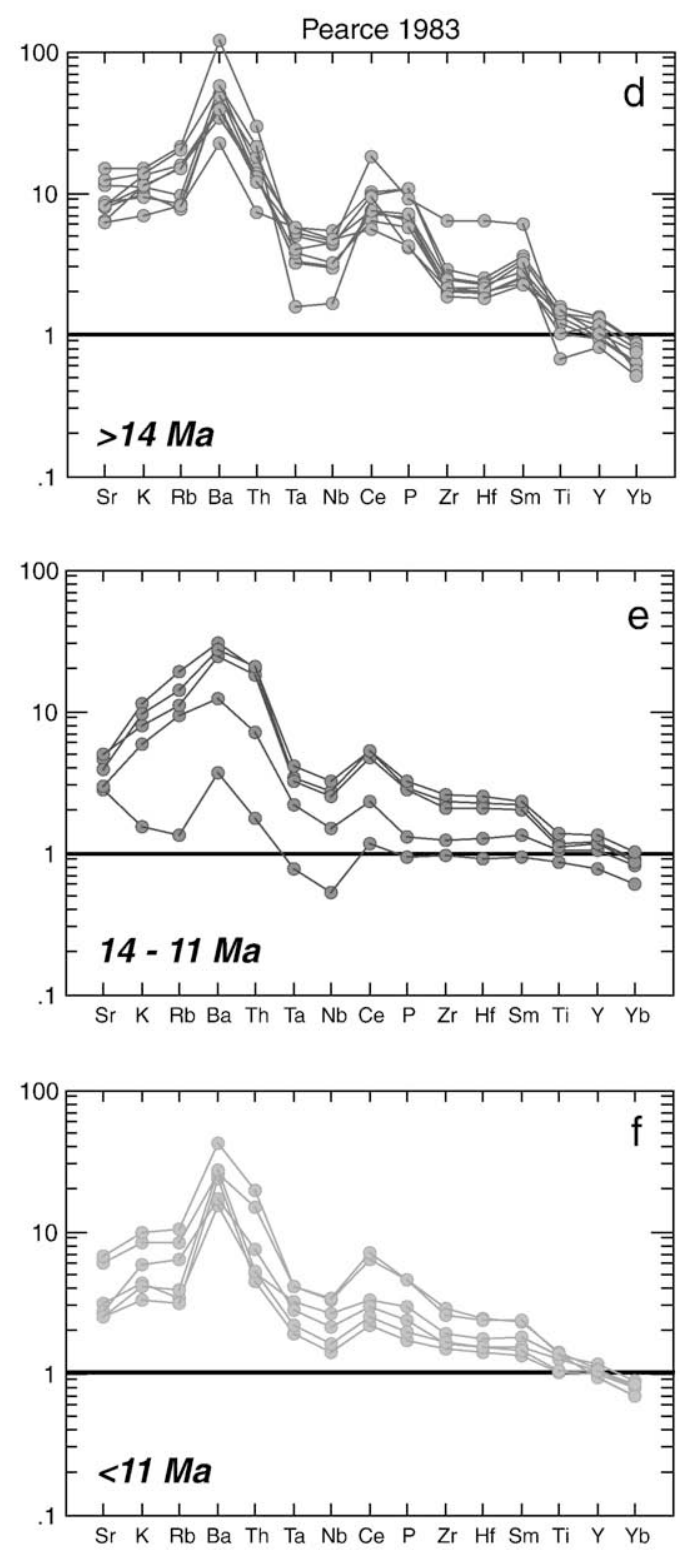

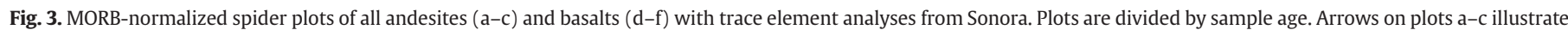
the progressive waning of the HFSE depletion and LILE enrichment after subduction ceased. 

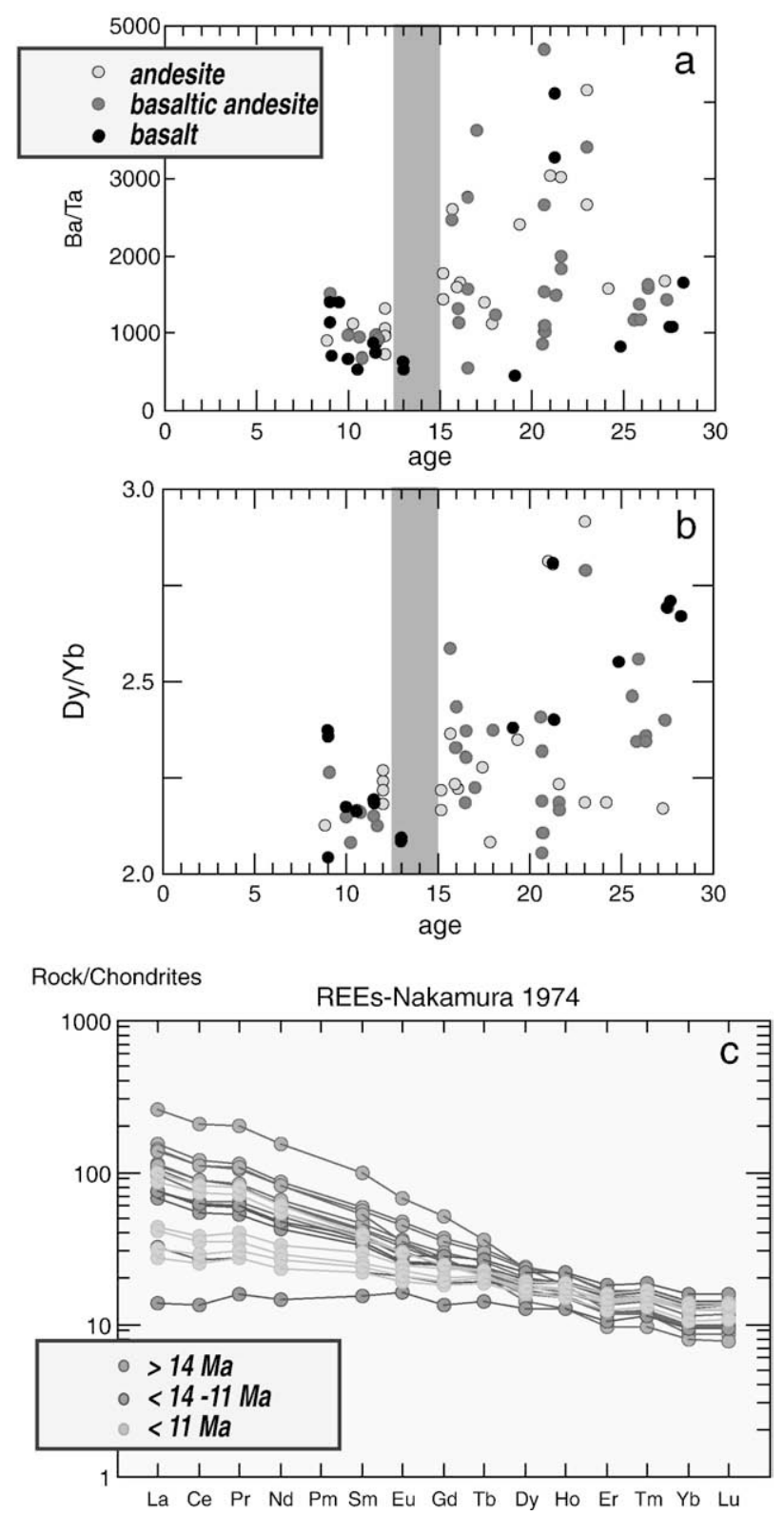

Fig. 4. Trace element ratios and REE plots for mafic samples from Sonora. Plots a. and b. are $\mathrm{Ba} / \mathrm{Ta}$ and $\mathrm{Dy} / \mathrm{Yb}$ ratios for all Sonoran andesites, basaltic andesites and basalts plotted against age. Gray box from 15-12.5 Ma denotes the approximate termination of subduction below Sonora. Plot c. is the REE concentration of all basalts color-coded by age.

Similar to the Colorado River extensional corridor in the Basin and Range (Gans and Bohrson, 1998), the cessation of eruptive activity in Sonora at $\sim 8$ Ma may have been a thermobarometric consequence of extensional fracturing and thinning of the upper crust.

Our interpretation of conductive reheating causing post-subduction volcanism in Sonora is consistent with that of Castillo (2008), who proposes post-subduction volcanism in Baja was the result of influx of Pacific asthenosphere after the cessation of subduction, which provided the thermal energy to melt mafic lower crust to form adakites (Atherton and Petfrod, 1993; Negrete-Aranda and CañónTapia, 2008) and the preexisting metasomatized mantle wedge, to produce bajaites and calc-alkaline magmas. Similarly, Vidal-Solano et al. (2008) conclude mid-Miocene volcanic rocks in Pinacate, Sonora, located northwest of our study area, formed during progressive thinning of the lithosphere and upwelling of asthenosphere, which they attribute to the formation of a slab window. As an
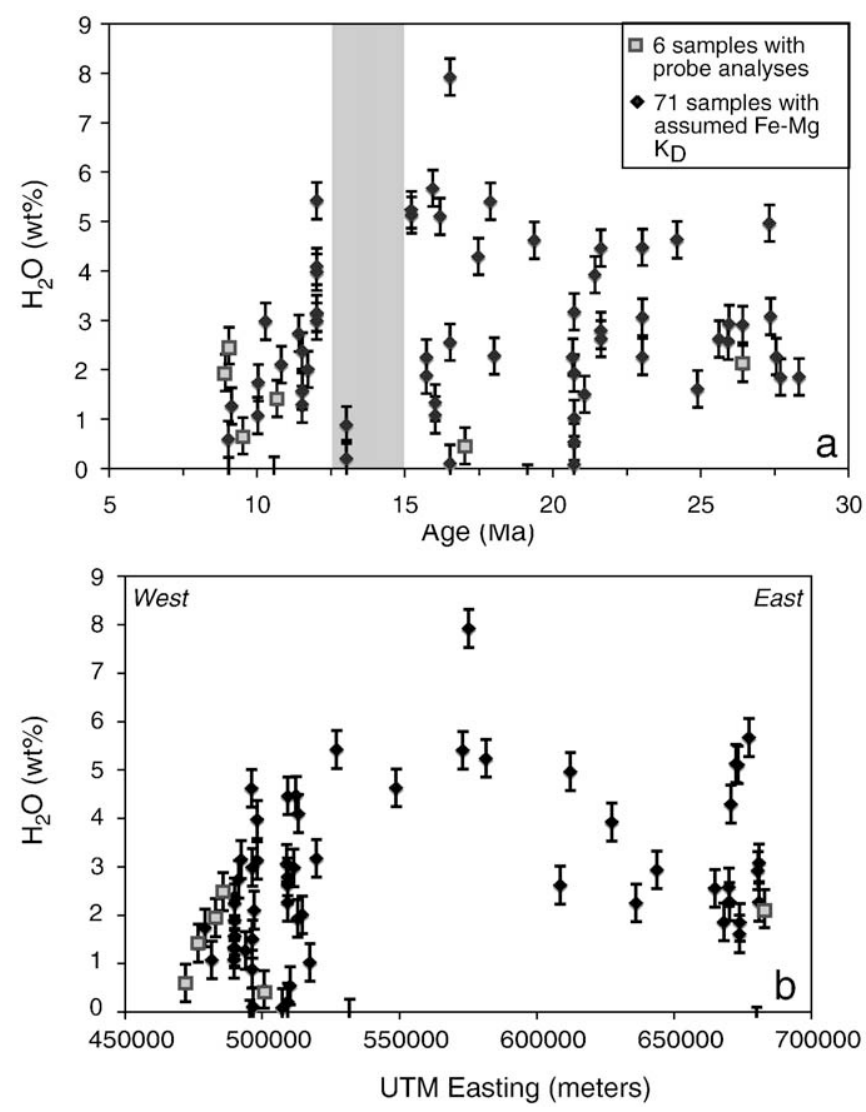

Fig. 5. Pre-eruptive $\mathrm{H}_{2} \mathrm{O}$-content for the 71 most mafic samples from Sonora plotted against time (a) and UTM easting (equivalent to longitude) (b). Pre-eruptive $\mathrm{H}_{2} \mathrm{O}$ content calculated using the olivine-clinopyroxene-plagioclase hydrometer of Sisson and Grove (1993). Square symbols denote subset of six samples for which $\mathrm{H}_{2} \mathrm{O}$-content was calculated using the phenocryst phase compositions rather than calculating the liquidus olivine composition, assuming a Fe-Mg $K_{\mathrm{D}}$ of 0.29 . Average error is approximately 0.39 wt.\% $\mathrm{H}_{2} \mathrm{O}$.

alternative, Cañón-Tapia and Walker (2004) suggest there can be a sufficient lag time (on the order of $\sim 10^{6}$ years), between deep melt generation and surface eruption, to allow magma reservoirs to act as time capsules that record the prior tectonic setting. Mantle and crustal heterogeneities, as well as local stress fields, all likely effect when and where magma erupts. Thus, Negrete-Aranda and Cañón-Tapia (2008) suggest in Baja and in similar structurally-active tectonic settings, we should reject the assumption that magmas have short subsurface residence times and thus the hypothesis that the type of volcanic rocks erupted is coupled with the present tectonic setting.

The overall similarity in syn- and post-subduction mafic volcanic rocks erupted in southern Sonora suggest either they were produced by conductive reheating of subduction-modified mantle or by long crustal residence times. A time lag of $2.5-4 \times 10^{6}$ years, between formation and eruption, is required to explain the subduction character of most post-subduction magmas in southern Sonora, which is at the uppermost limit of residence times predicted by Cañón-Tapia and Walker (2004). Unlike the central and southern regions of the Baja peninsula, Sonora exhibits none of the traditional compositions of volcanism associated with extensional terrains, such as alkalic or tholeiitic basalt or bimodal rhyolitic and basaltic volcanism (Gastil et al., 1979; Hausback, 1984; Sawlan and Smith, 1984; Saunders et al., 1987; Bigioggero et al., 1995; Luhr et al., 1995). However, the presence of $<12.5$ Ma relatively anhydrous lavas in coastal Sonora suggest that not all post-subduction magmas acted as "time capsules" (Fig. 5b). Thus we favor a model where extension and asthenospheric upwelling caused melting of previously metasomatized mantle following subduction in southern Sonora, and recognize 


\section{a $\operatorname{SYN}-S U B D U C T I O N(>15 \mathrm{Ma})$}

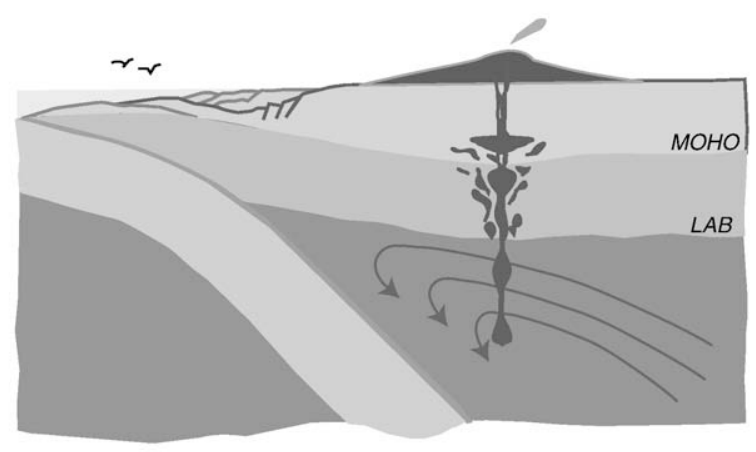

\section{b POST-SUBDUCTION (12.5-8 Ma)}

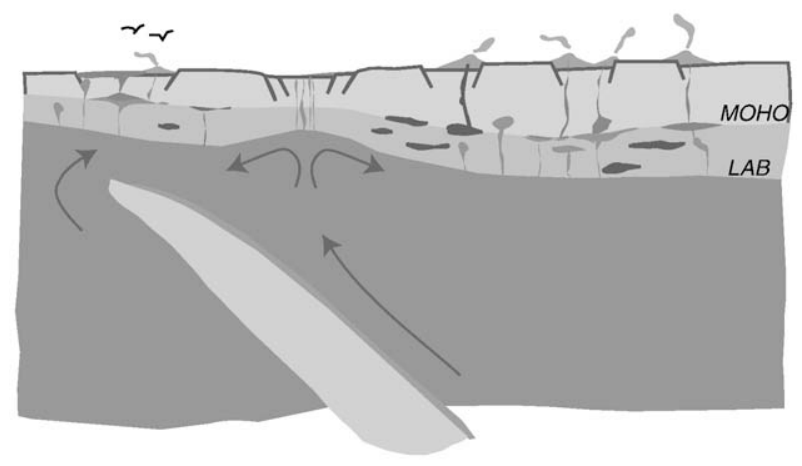

Fig. 6. Cartoon cross-section through the Gulf Extensional Province during subduction (a) and following the tectonic transition to oblique extension (b). Syn-subduction volcanism is the product of typical flux melting of hydrated mantle wedge peridotite. Post-subduction magmatism $(<12.5 \mathrm{Ma})$ likely formed when proto-Gulf extension triggered conductive reheating and/or decompression melting of reservoirs of metasomatized mantle peridotite.

the potential that some magma reservoirs may have resided in the crust for on the order of $10^{6}$ years before erupting.

\section{Conclusion}

The age-constrained major and trace element analyses presented in this study represent the most comprehensive geochemical database for Cenozoic volcanism in Sonora. Mafic volcanic rocks erupted in Sonora from 27 to $8 \mathrm{Ma}$ have continental arc signatures and appear to be derived from the metasomatized sub-arc mantle below Sonora. Samples erupted during subduction (ca. 27-15 Ma) likely formed via typical flux melting of hydrated mantle wedge peridotite and subsequent fractionation processes in the crust. The residual arc trace element signatures and low pre-eruptive $\mathrm{H}_{2} \mathrm{O}$-content of samples erupted after subduction ceased (ca. 12.5-8 Ma) suggests the time required to completely erase the subduction signature from the sub-arc mantle was greater than the 4 million years of volcanism that post-dated subduction in Sonora. Post-subduction samples likely represent the remobilization of subduction-altered mantle lithosphere triggered by crustal extension in Sonora or had significant residence times in the crust. Southern Sonora is unlike central and southern regions of Baja, which demonstrate a change in the average composition of magmas erupted after subduction ceased ca. $11 \mathrm{Ma}$. Instead Sonora exhibits only subtle and protracted ( $>4$ m.y.) changes in the geochemical record. Therefore although both Baja and Sonora experienced an abrupt (2.5 m.y.) change in tectonic setting, they exhibit very different responses in the geochemical record. This study demonstrates the need for caution for interpreting geochemical data in the absence of plate reconstructions.

\section{Acknowledgements}

This work was funded by the National Science Foundation. We thank J. Roldan-Quintana for logistical support, WSU GeoAnalytical Lab for analytical assistance, I. MacMillan for field support and C. Snow and B. Leeman for review comments on an earlier version of this paper.

\section{Appendix A. Supplementary data}

Supplementary data associated with this article can be found, in the online version, at doi:10.1016/j.jvolgeores.2009.06.014.

\section{References}

Aranda-Gomez, J.J., McDowell, F.W., 1998. Paleogene extension in the southern Basin and Range Province of Mexico; syndepositional tilting of Eocene red beds and Oligocene volcanic rocks in the Guanajuato mining district. International Geology Review 40 (2), 116-134.

Arculus, R.J., Johnson, R.W., 1981. Island-arc magma sources - a geochemical assessment of the roles of slab-derived components and crustal contamination. Geochemical Journal 15 (3), 109-133.

Atherton, M.P., Petford, N., 1993. Generation of sodium-rich-magmas from newly underplated basaltic crust. Nature 362, 144-146.

Atwater, T., 1970. Implications of plate tectonics for the Cenozoic tectonic evolution of western North America. Geological Society of America Bulletin 81 (12), 3513-3535.

Atwater, T., 1989. Plate tectonic history of the northeast Pacific and western North American. In: Winterer, E.L., et al. (Ed.), The Eastern Pacific Ocean and Hawaii: Geology of North America volume N. Geological Society of America, Boulder, Colorado, pp. 21-71.

Bigioggero, B., Chiesa, S., Zanchi, A., Montrasio, A., Vezzoli, L., 1995. The Cerro Mencenares volcanic center, Baja California Sur; source and tectonic control on postsubduction magmatism within the Gulf Rift. Geological Society of America Bulletin 107 (9), 1108-1122.

Blair, K.D., personal communication, 2006.

Blair, K., Gans, P.B., 2003. Stratigraphy of the Sahuaripa Basin and preliminary comparison to the Rio Yaqui Basin, east-central Sonora, Mexico. Abstracts with Programs-Geological Society of America 35 (4), 27.

Blair, K., Gans, P.B., 2005. The Sahuaripa Basin: episodic Cretaceous to Mid-Miocene extension and magmatism in the Northwestern Mexican Basin and Range. Abstracts with Programs-Geological Society of America 37 (7), 480.

Castillo, P.R., 2008. Origin of the adakite-high-Nb basalt association and its implications for postsubduction magmatism in Baja California, Mexico. Geological Society of America Bulletin 120 (3/4), 451-462.

Cañón-Tapia, E., Walker, G.P.L., 2004. Global aspects of volcanism: the perspectives of "plate tectonics" and "volcanic systems.". Earth Science Reviews 66, 163-182.

Chayes, F., 1964. A Petrographic distinction between Cenozoic Volcanics in and around the Open Oceans. Journal of Geophysical Research 69 (8), 1573-1588.

Christiansen, R.L., Lipman, P.W., 1972. Cenozoic Volcanism and Plate-Tectonic Evolution of the Western United States. II Late Cenozoic. Philosophical Transactions of the Royal Society of London. Series A, Mathematical and Physical Sciences 271 (1213) 249-284.

Daly, R.A., 1933. Igneous Rocks and the Depths of the Earth. McGraw-Hill, New York. 598 pp. Dickinson, W.R., Snyder, W.S., 1979. Geometry of triple junctions related to San Andreas Transform. Journal of Geophysical Research 84 (NB2), 561-572.

Dupuy, C., Dostal, J., Marcelot, G., Bougault, H., Joron, J.L., Treuil, M., 1982. Geochemistry of basalts from Central and Southern New Hebrides Arc - implications for their source rock compositions. Earth and Planetary Science Letters 60 (2), 207-225.

Gans, P.B., 1997. Large-magnitude Oligo-Miocene extension in southern Sonora; implications for the tectonic evolution of Northwest Mexico. Tectonics 16 (3) 388-408.

Gans, P.B., personal communication, 2009.

Gans, P.B., Bohrson, W.A., 1998. Suppression of volcanism during rapid extension in the Basin and Range Province, United States. Science 279, 66-68.

Gans, P.B., Wong, M., MacMillan, I., Blair, K.D., Roldan, J., Till, C.B., Herman, S., 2006 Magmatic-tectonic interactions in the Gulf extensional province: insights from the Sonoran margin. Proceedings, MARGINS Workshop: Lithospheric Rupture in the Gulf of California - Salton Trough Region. http://www.rcl-cortez.wustl.edu/ Presentations.html.

Gastil, R.G., Phillips, R.P., and Allison, E.C., 1975. Reconnaissance geology of the state of Baja California. Geological Society of America Memoir Map 140, scale 1; 250,000.

Gastil, G., Krummenacher, D., Minch, J., 1979. The record of Cenozoic volcanism around the Gulf of California. Geological Society of America Bulletin 90 (9), I839-I857.

Gastil, R.G., Neuhaus, J., Cassidy, M., Smith, J.T., Ingle Jr., J.C., Krummenacher, D., 1999 Geology and paleontology of southwestern Isla Tiburon, Sonora, Mexico. Revista Mexicana de Ciencias Geologicas 16 (1), 1-34.

Gill, J.B., 1981. Orogenic Andesites. Springer-Verlag, NewYork. 390 pp. 
Grove, T.L., Parman, S.W., Bowring, S.A., Price, R.C., Baker, M.B., 2002. The role of an $\mathrm{H}_{2} \mathrm{O}$ rich fluid component in the generation of primitive basaltic andesites and andesites from the Mt. Shasta region, N California. Contributions to Mineralogy and Petrology 142, 375-396.

Hausback, B.P., 1984. Cenozoic volcanic and tectonic evolution of Baja California Sur Mexico: Field Trip Guidebook - Pacific Section, Society of Economic Paleontologists and Mineralogists, vol. 39, pp. 219-236.

Herman, S.W., Gans, P.B., 2005. Large scale vertical axis rotations in coastal Sonora; evidence for transtensional proto-Gulf deformation. Abstracts with ProgramsGeological Society of America 37 (7), 68.

Kelemen, P.B., Yogodzinski, G.M., Scholl, D.W., 2003. Along-strike variations in the Aleutian Island arc: genesis of high Mg\# andesite and implications for continental crust. In: Eiler, J. (Ed.), Inside the Subduction Factory. American Geophysical Union, Washington DC, pp. 223-276.

Kovács, I., Szabó, C.S., 2008. Middle Miocene volcanism in the vicinity of the Middle Hungarian zone: evidence for an inherited enriched mantle source. Journal of Geodynamics 45, 1-17.

Le Bas, M.J., Le Maitre, R.W., Woolley, A.R., 1992. The construction of the total alkali-silica chemical classification of volcanic rocks. Mineralogy and Petrology 46, 1-22.

Lonsdale, P., 1991. Structural patterns of the Pacific floor offshore of Peninsular California. In: Dauphin, J.P., Simoneit, B.R.T. (Eds.), The Gulf and Peninsular Province of the Californias: AAPG Memoir, vol. 47, pp. 87-125.

Lonsdale, P., 2006. The opening of the Gulf of California Trough within the North American Cordillera. Abstracts with Programs - Geological Society of America Specialty Meeting, Backbone of the Americas, Mendoza, Argentina, p. 82.

Luhr, J.F., Aranda-Gomez, J.J., Housh, T.B., 1995. San Quintin volcanic field, Baja California Norte, Mexico: geology, petrology and geochemistry. Journal of Geophysical Research 100 (B7), 10353-10380.

MacMillan, I., personal communication, 2006

MacMillan, I., Gans, P.B., Roldan, J., 2003. Voluminous mid-Miocene silicic volcanism and rapid extension in the Sierra Libre, Sonora, Mexico. Abstracts with Programs Geological Society of America 35 (4), 26.

MacMillan, I., Gans, P.B., Till, C.B., 2005. Tectonic implications of the volcanic and structural history of the Sierra Santa Ursula, Sonora Mexico. Abstracts with Programs-Geological Society of America 37 (4), 64.

Marshall, P., 1912. Oceania, Handbuch der Regionalen Geologie, vol. 2. C. Winter, Heidelberg, pp. 1-36.

McDowell, F.W., Clabaugh, S.E., 1979. Ignimbrites of the Sierra Madre Occidental and their relation to the tectonic history of western Mexico. Special Paper-Geological Society of America 180, 113-124.

McDowell, F.W., Roldan Quintana, J., Amaya Martinez, R., 1997. Interrelationship of sedimentary and volcanic deposits associated with Tertiary extension in Sonora, Mexico. Geological Society of America Bulletin 109 (10), 1349-1360.

Mora-Klepeis, G., McDowell, F.W., 2004. Late Miocene calc-alkalic volcanism in northwestern Mexico: an expression of rift or subduction-related magmatism? Journal of South American Earth Sciences 17, 297-310.

Negrete-Aranda, R., Cañón-Tapia, E., 2008. Post-subduction volcanism in the Baja California Peninsula, Mexico: the effects of tectonic reconfiguration in volcanic systems. Lithos 102, 392-414.

Oskin, M., Stock, J., 2003. Cenozoic volcanism and tectonics of the continental margins of the upper Delfin Basin, northeastern Baja California and western Sonora. In: Johnson, S.E., et al. (Ed.), Tectonic Evolution of Northwestern Mexico and the Southwestern USA: Geological Society of America Special Paper, vol. 374, pp. 421-438. Boulder, Colorado.

Pearce, J.A., Cann, J.R., 1971. Ophiolite origin investigated by discriminant analysis using Ti, Zr and Y. Earth and Planetary Science Letters 12, 339.

Pearce, J.A., Cann, J.R., 1973. Tectonic setting of basic volcanic rocks determined using trace element analyses. Earth and Planetary Science Letters 19, 290-300.

Pearce, J.A., Peate, D.W., 1995. Tectonic implications of the composition of volcanic arc magmas. Annual Reviews of Earth and Planetary Sciences 23, 251-285.

Perfit, M.R., Gust, D.A., Bence, A.E., Arculus, R.J., Taylor, S.R., 1980. Chemical characteristics of island-arc basalts: implications for mantle sources. Chemical Geology 30, 227-256.

Saunders, A.D., Rogers, G., Marriner, G.F., Terrell, D.J., Verma, S.P, 1987. Geochemistry of Cenozoic volcanic rocks, Baja California, Mexico; implications for the petrogenesis of post-subduction magmas. Journal of Volcanology and Geothermal Research 32 (1-3), 223-245.

Sawlan, M.G., 1991. Magmatic evolution of the Gulf of California rift. In: Dauphin, J.P., Simoneit, B.R.T. (Eds.), The Gulf and Peninsular Province of the Californias: American Association of Petroleum Geologists Memoir, vol. 47, pp. 301-369. Tulsa, Oklahoma.

Sawlan, M.G., Smith, J.G., 1984. Late Cenozoic volcanic suites in northern Baja California Sur, Mexico; their relation to subduction and rifting along the Baja California Peninsula. Abstracts with Programs-Geological Society of America 16 (6), 645.

Sisson, T.W., Grove, T.L., 1993. Experimental investigations of the role of $\mathrm{H}_{2} \mathrm{O}$ in calcalkaline differentiation and subduction zone magmatism. Contributions to Mineralogy and Petrology 113 (2), 143-166.

Spera, F.J., Bohrson, W.A., Till, C.B., Ghiorso, M.S., 2007. Partitioning of trace elements among coexisting crystals, melt, and supercritical fluid during isobaric crystallization and melting. American Mineralogist 92 (11-12), 1881-1898.

Stock, J.M., Hodges, K.V., 1989. Pre-Pliocene extension around the Gulf of California, and the transfer of Baja California to the Pacific Plate. Tectonics 8 (1), 99-115.

Valencia-Moreno, M., Ruiz, J., Barton, M.D., Patchett, P.J., Zuercher, L., Hodkinson, D.J., Roldan-Quintana, J., 2001. A chemical and isotopic study of the Laramide granitic belt of northwestern Mexico; identification of the southern edge of the North American Precambrian basement. Geological Society of America Bulletin 113 (11), $1409-1422$.

Vidal-Solano, J.R. Demant, A., Paz Moreno, F.A. Lapierre, H, Ortega-Rivera, M.A., Lee, J.K.W. 2008. Insights into the tectonomagmatic evolution of NW Mexico: geochronology and geochemistry of the Miocene volcanic rocks from the Pinacate area, Sonora. Geological Society of America Bulletin 120 (5/6), 691-708.

Weigand, P.W., Savage, K.L., Nicholson, C., 2002. The Conejo Volcanics and other Miocene volcanic suites in southwestern California. In: Barth, A. (Ed.), Contributions to Crustal Evolution of the Southwestern United States: Geological Society of America Special Paper, vol. 365, pp. 187-204. Boulder, Colorado.

Wong, M.S., Gans, P.B., 2003. Tectonic implications of early Miocene extensional unroofing of the Sierra Mazatán metamorphic core complex, Sonora, Mexico. Geology 31 (11), 953-956.

Wong, M.S., Gans, P.B., 2008. Geologic, structural, and thermochronologic constraints on the tectonic evolution of the Sierra Mazatan core complex, Sonora, Mexico: new insights into metamorphic core complex formation. Tectonics 27, 1-31. 